tum given intramuscularly in case 2 would not have been effective at the time of the crisis. Both patients were breathing spontaneously and would have been hypercapnoeic. The insertion of cocaine and adrenaline paste and almost immediate use of instruments in the nose in a patient with high catecholamine concentrations, a respiratory acidosis, and circulating halothane is a recipe for arrhythmias.

Although the anaesthetic technique in case 3 cannot be faulted, the cocaine and adrenaline paste was inserted into the nose 25 minutes after induction of anaesthesia, at which time surgery can be presumed to have started and the nasal mucous membrane had been disrupted. The insertion of paste into the nose in these circumstances and the immediate use of instruments would force some of the mixture rapidly into the circulation, with the inevitable likelihood of serious arrhythmias.

The usual practice when using cocaine and adrenaline paste is to place it into the nose $10-15$ minutes before induction of anaesthesia, during which time the patient's pulse is monitored Experience shows that a certain period is required to obtain the full benefit of the paste in terms of vascular constriction and shrinking of the nasal mucosa. Although cocaine alone will produce some vasoconstriction and mucosal shrinking, the operating conditions are undoubtedly better when cocaine is combined with adrenaline.

The complications associated with the use of any drug can be avoided by not exceeding the correct dose and by giving the drug in the correct manner. When an interaction with other drugsfor example, anaesthetic agents-might be expected the anaesthetic technique used must take this into account.

On the basis of the three cases reported, the authors' condemnation of the use of cocaine and adrenaline paste is unjustified and the suggestion that its continued use may have medicolegal implications is unnecessarily alarmist.

PD M ELLIS roat surgeo B R WILKEY

Addenbrooke's Hospital Consultant anaesthetis Cambridge CB2 2QQ

1 Nicholson KEA, Rogers JEG. Cocaine and adrenaline paste: a fatal combination? BMF 1995;311:250-1. (22 July.)

\section{Inserting central venous catheters}

\section{Case for angiographically guided placement is not proved}

EDrToR,-Andy Adam's editorial suggesting that long term central venous catheters (Hickman lines) should be placed only by interventional radiologists contains many errors that seriously weaken his argument." His description of "traditional" surgical placement is incorrect: the lines are placed into the internal jugular vein or, less commonly, the cephalic vein and not, as he suggests, into the subclavian vein. The risks of arterial puncture in patients with coagulopathies is overstated, simply because coagulopathies should always be corrected before insertion of a central venous catheter, and the use of imaging does not exempt the operator from this requirement.

Adam states that the rate of arterial puncture is reduced when imaging is used. This is offset by the observation that the only death associated with placement of a catheter in one of the series that Adam cites in which catheters were placed by interventional radiologists occurred after arterial puncture in a patient with a coagulopathy. ${ }^{2}$ Adam also states that the rate of misplaced catheters in series in which imaging was not used was $6 \%{ }^{3}$ This is not strictly accurate as the study showed that use of ultrasound imaging to locate the subclavian vein conferred no advantage over use of anatomical landmarks. The $6 \%$ rate of misplacement related to the whole study, including those patients in whom ultrasound imaging was used.

Imaging guidance does not "allow the procedure to be carried out under local anaesthesia and light sedation" as Adam suggests, as both surgical and blind percutaneous techniques can be undertaken this way. The use or lack of use of imaging has no bearing on the type of anaesthesia used. Moreover, it is the use of local anaesthesia and light sedation coupled with ingeniously designed catheters that converts placement of a central venous catheter from a surgical procedure to an outpatient procedure performed in a treatment room, not the skills of interventional radiologists.

Cardiologists, nephrologists, anaesthetists, intensivists, paediatricians, and many other groups are skilled in the techniques of venepuncture and the use of guidewires. In addition, small, portable ultrasound imaging devices specifcally designed for imaging vessels and introducing catheters are available. With these devices the subclavian artery and vein can easily be distinguished and the vein punctured. Clearly, the skills required to place these lines are not unique to interventional radiologists.

Angiography facilities may not be as accessible as Adam suggests: they may not even be in the same hospital as the patient. It might be possible to show a cost benefit when placement in an angiography suite is compared with surgical placement, but we think that placement of routine catheters on the ward under local anaesthesia and sedation is the most economical option. Our success rate is $96 \%$; we use percutaneous unguided techniques. There is no evidence that guided insertion is safer, cheaper, or more effective. We suggest that the "time for a new look" has not yet come.

DUNCAN YOUNG Clinical reader in anaesthetics MICHAEI KETTLEWELI Consultant surgeon HELEN HAMILTON Nuffield Department of Anaesthetics, HELEN HAMILTON
Clinical nurse specialist ohn Radcliffe Hospital Oxford OX3 9DU

1 Adam A. Insertion of long term central venous catheters: time for a new look. $B M 7$ 1995;311:341-2. (5 August.)

2 Robertson LJ, Mauro MA, Jaques PF. Radiologic placement of Hickman catheters. Radiology 1989;107:1007-9.

3 Mansfield PF, Hohn DC, Fornage BD, Gregurich MA, Ota DM. Complications and failures of subclavian-vein catheterization. N Engl f Med 1994;331:1735-8.

\section{Open technique has lower evidence of complications}

EDrror,-We disagree with Andy Adam's comments regarding the drawbacks of an open surgical technique for placing long term central venous catheters. It is our practice to place such catheters by using an open cutdown technique under local anaesthesia. We mainly use the cephalic vein, with fluoroscopic screening to ensure correct positioning. With this technique more than 800 catheters have been inserted over the past 10 years, for parenteral nutrition, antibiotics, and chemotherapy. The incidence of pneumothorax and injury to vessels at the root of the neck has been zero. These types of complications are seen when blind puncture techniques are used and were one reason for our change to an open technique. The bulk of our practice is the treatment of very sick patients with intestinal failure, who would tolerate such complications badly. Using our method, over the past 12 months we have failed to achieve central venous access in only two patients. We regard the open technique as a safe and reliable means of venous access that is well tolerated by our patients.

Adam seems to dismiss the surgical technique on the basis of a single study. ${ }^{2}$ Without a systematic appraisal of the literature there is a risk that important evidence of effectiveness may be missed, and this may bias the conclusions that are drawn. ${ }^{3}$ The references cited in the editorial do not include any randomised controlled trials, possibly because none exist. We would like to see evidence from more rigorous trials before we accept that one method is superior.

To solve the problem of lack of evidence regarding the techniques we recommend a systematic review of the literature. If doubt still existed then a randomised controlled trial comparing both methods of inserting catheters, including a cost analysis, would be appropriate.

The more important issue to be addressed is not the technique of inserting catheters but their subsequent care. Life threatening complications of central lines-namely, sepsis and central venous thrombosis-occur because of inadequate care. Patients should not be discharged until they or a designated person are competent to look after the line. This requires a great deal of professional skill and is a major factor in the success of the intestinal failure unit at Hope Hospital.

$\begin{array}{rr}\text { D M RICHARDS } & \text { N A SCOTT } \\ \text { Tutor in surgery } & \text { Consultant general surgeon } \\ \text { J HILL } & \text { J BANCEWICZ } \\ \text { Reader in surgery } \\ \text { MILES IRVING } \\ \text { Senior registrar in surgery } & \text { Professor of surgery }\end{array}$

Department of Surgery,

Salford M6 8HD

1 Adam A. Insertion of long term central venous catheters: time for a new look. $B M F$ 1995;311:341-2. (5 August.)

David SJ, Thompson JS, Edney JA. Insertion of Hickman catheters in total parenteral nutrition: a prospective study of 200 consecutive patients. Am f Surg 1984;50:673-6.

3 Mulrow CD. Systematic review: rationale for systematic reviews. $B M F$ 1994;309:597-9.

\section{Difficulties are rare and do not correlate with lifespan of catheter}

EDITOR,-We strongly support Andy Adam's view that imaging guidance and experience are necessary for the placement of Hickman-type catheters. ${ }^{1}$ The first surgical insertions of Hickman catheters at the Royal Marsden Hospitals were described in $1979,{ }^{2}$ but over the past decade the anaesthetists have developed a comprehensive percutaneous venous access service, and they currently insert over 1000 lines a year. Dedicated theatre lists of up to 10 patients are eminently suitable for consultants to teach the technique to trainee anaesthetists.

Oncology patients are carefully worked up preoperatively: clotting function is optimised and the suitability of the site of insertion assessed. In complicated cases, in which there is chest disease or venous thrombosis, radiological investigations are performed with the full cooperation of the radiology department. The lines are inserted with a combination of good quality sedation for adults, general anaesthesia for children, safe doses of local anaesthetics, oxygen treatment, and full monitoring. Although serious complications are rare, full resuscitation back up is essential, as are recovery facilities after any procedure that requires sedation. ${ }^{3}$ The insertion technique has been described by Stacey et al..$^{4}$ Fluoroscopic screening is used for each step of the procedure. A recent internal audit showed that an open venous cutdown technique was required in only one in 1494 insertions and may be necessary for paediatric patients and patients with a refractory coagulopathy.

The lifespan of the catheter is of vital importance after insertion; in two consecutive audits at the Royal Marsden Hospital it has been found to be 91 days in 545 mixed oncology patients and 122 days in 949 patients with gastrointestinal malignancy. In the second audit a relation was sought between difficulties in inserting the catheter 